\title{
Dual-modal tracking of transplanted mesenchymal stem cells after myocardial infarction
}

This article was published in the following Dove Press journal:

International Journal of Nanomedicine

16 April 201 I

Number of times this article has been viewed

Yefei $\mathrm{Li}^{*}$

Yuyu Yao*

Zulong Sheng

Yanxiaoxiao Yang

Genshan $\mathrm{Ma}$

Department of Cardiology,

Zhongda Hospital, Medical School of Southeast University, Nanjing,

Jiangsu, China

*These two authors contributed equally to this work.
Correspondence: Genshan Ma Department of Cardiology, Zhongda Hospital, Medical School of Southeast University, No 87, Dingjiaqiao, Nanjing, Jiangsu, 210009 , People's Republic of China Tel +86 2583272595

Fax +862583272038

Email magenshan@hotmail.com
Purpose: Results for implantation efficiency and effective improvement of cardiac function in the field of mesenchymal stem cells (MSCs) are controversial. To attempt to clarify this debate, we utilized magnetic resonance imaging (MRI) and near-infrared optical imaging (OI) to explore the effects of different delivery modes of mesenchymal stem cells on cell retention time and cardiac function after myocardial infarction (MI).

Methods: Rat MSCs were labeled with superparamagnetic iron oxide nanoparticles and 1, 1'-dioctadecyl-3,3,3',3'-tetramethylindodicarbocyanine, 4-chlorobenzenesulfonate salt (DiD) for noninvasive cell tracking in a rat MI model. Rats underwent coronary artery ligation and were randomized into three experimental groups: intravenous (IV), intramyocardial (IM), and a control group. The first two groups referred to the route of delivery of the transplanted duallabeled MSCs; whereas the control group was given an IV injection of serum-free medium one day post-MI. Cellular engraftment was determined 1 day and 7 days post cell delivery by measuring the iron and optical signals in explanted organs. Prussian blue staining and fluorescent microscopy were performed on histological sections for iron and DiD, respectively. Cardiac function was measured by echocardiography on day 7 .

Results: The cardiac function of the IM group increased significantly compared to the IV and control groups at day 7. In the IM group, labeled cells were visualized in the infracted heart by serial MRI, and the intensity by OI was significantly higher on day 1. In the IV group, the heart signals were significantly attenuated by dual-modal tracking at two time points, but the lung signals in OI were significantly stronger than the IM group at both time points.

Conclusion: IM injection of MSCs increased cell engraftment within infarcted hearts and improved cardiac function after MI. However, IV infusion has a low efficacy due to the cell trapping in the lung. Therefore, direct injection may provide an advantage over IV, with regard to retention of stem cells and protection of cardiac function.

Keywords: stem cell tracking, superparamagnetic iron oxide, DiD, cardiac function, myocardial infarction

\section{Introduction}

Myocardial infarction (MI) is one of the leading causes of death worldwide with an increasing morbidity and mortality of heart failure after MI. ${ }^{1}$ A loss of functional cardiomyocytes comprises the cellular basis for cardiac dysfunction and heart failure. Stem cell-based repletion of scarred myocardial tissue and regeneration of functional cardiomyocytes have been proposed as a potential treatment for the infarcted heart. ${ }^{2}$ A number of major clinical trials have reported beneficial results, including improved cardiac contractile function and suppression of left ventricle remodelling. ${ }^{3}$ However, some researchers have shown only modest, negative, or merely a transient effect on 
cardiac function. ${ }^{4-7}$ This indicates that factors such as cell type, number of transplanted cells, optimal timing, cell delivery route, and the mechanism of action remain to be explored.

Bone marrow-derived mesenchymal stem cells (MSCs) have the multipotent capability to differentiate under appropriate conditions into chondrocytes, tenocytes, myocytes, neurons, cardiomyocyte-like cells, vascular smooth muscle cells, and endothelial cells. Together this points to a high degree of plasticity, ${ }^{8,9}$ which has been used successfully in cell transplantation without immune rejection. ${ }^{10}$ There are, however, controversial results regarding implantation efficiency and improved function in cardiac cell therapy. 3,7,11,12 These discrepancies may arise from the different modes of cell delivery. The appropriate delivery approach for MSC treatment post-MI has not been clarified. Intravenous infusion and intramyocardial injection are the current predominant stem cell delivery routes for the infarcted heart of small animals. ${ }^{13,14}$ Cell delivery by intravenous infusion has the advantage of simple administration, but Carr et al reported it could not ameliorate cardiac dysfunction. ${ }^{15}$ In contrast, the intramyocardial route has the associated disadvantages of being a highly invasive operation and requires a limited dose. ${ }^{16,17}$

Magnetic resonance imaging (MRI) possesses high spatial resolution, but less sensitivity in stem cell tracking. However, near-infrared (NIR) optical imaging (OI) has proven highly sensitive for in vivo cell tracking. We took advantage of these two imaging modalities for in vivo tracking of transplanted MSCs. This study was designed to compare the efficiency of MSC therapy through two delivery routes using MR and NIR optical dual-modality imaging techniques to determine the appropriate route of cell delivery for clinical use.

\section{Materials and methods Experimental reagents}

The following reagents were used in this study: fetal bovine serum (FBS; Gibco, Carlsbad, CA), Dulbecco's modified Eagle's medium (DMEM; Hyclone, Logan, UT), trypsin (Beyotime, Beijing, China), superparamagnetic iron oxide (SPIO; Feridex, Resovist, Berlin, Germany), 1,1'-dioctadecyl-3,3,3',3'-tetramethylindodicarbocyanine, 4-chlorobenzenesulfonate salt (DiD; Invitrogen, Carlsbad, CA), 3-(4,5-dimethylthiazol-2-yl)-2,5-diphenyl tetrazolium bromide (MTT; Sigma, St Louis, MO), dimethyl sulfoxide (DMSO; Sigma), Prussian blue staining kit (Sigma), ED-1 antibody (Abbiotec, San Diego, CA).

\section{MSC culture and labeling}

Male Sprague Dawley (SD) rat femurs and tibias were isolated under sterile conditions and bone marrow was flushed out using DMEM medium. ${ }^{18}$ Subsequently, single-nucleated cells were culture-expanded for three passages in vitro. Magnetic labeling of the MSCs was achieved by 24-hour incubation in culture medium containing a ferumoxides injectable solution $(25 \mu \mathrm{g}$ Fe per $\mathrm{mL}$ ) mixed with poly-L-lysine (PLL; $37.5 \mathrm{ng} / \mathrm{mL}$; average molecular weight $275 \mathrm{kDa}$ ) 1 hour before cell incubation. ${ }^{19}$ After SPIO labeling, cells were trypsinized and then resuspended at a concentration of $1 \times 10^{6} \mathrm{cell} / \mathrm{s} / \mathrm{mL}$ in serum-free DMEM medium. Sterile DiD cell-labeling stock solution was added to the cell suspension $(5 \mu \mathrm{L}$ per $\mathrm{mL})$ followed by incubation for 20 minutes at $37^{\circ} \mathrm{C}$. After labeling, cells were washed three times with warm medium $\left(37^{\circ} \mathrm{C}\right)$ to remove unbound DiD. Subsequently, the labeled cells were assessed by cell proliferation assay and labeling efficiency measurements or prepared for cell transplantation.

\section{Cell proliferation test}

MTT assays were performed to monitor cell proliferation in the labeled and unlabeled cells. The labeled and unlabeled cells were seeded into 96 flat-bottom well plates with $2 \times 10^{3}$ or $1 \times 10^{3}$ cells per well for measurements on day 3 or day 7 post-seeding, respectively. After 3 or 7 days cultured in complete medium, $20 \mu \mathrm{L}$ MTT solution $(5 \mathrm{mg} / \mathrm{mL})$ was added to each well and incubated for 4 hours at $37^{\circ} \mathrm{C}$. The medium was removed and $150 \mu \mathrm{L}$ DMSO added to each well, and quantified by measuring the optical density (OD) at a wavelength of $490 \mathrm{~nm}$ with the Bio-Rad microplate reader (Bio-Rad Laboratories, Hercules, CA).

\section{Myocardial infarction model and cell transplantation}

All animal studies were approved by the Institutional Animal Care and Use Committee of Southeast University. The recipient female SD rats (weight $80-100 \mathrm{~g}$ ) were anesthetized with pentobarbital (30 mg/kg given intraperitoneally), intubated and ventilated at 60 breaths per minute. The left anterior descending coronary (LAD) artery was ligated proximally with 8-0 silk suture via a left thoracotomy incision. Thirty-six rats with a left ventricular ejection fraction (LVEF) below $40 \%$ as measured by echocardiography were used in this study. Rats were evenly randomized into control group, intravenous (IV) infusion group and intramyocardial (IM) injection group. Each rat among cell transplantation groups (IV or IM) received $1 \times 10^{6}$ dual-labeled MSCs in $100 \mu \mathrm{L}$ of serum-free medium 1 day after operation by IV or direct 
IM injection. In the control group, rats received the $100 \mu \mathrm{L}$ of serum-free medium through an IV injection. Each of the three groups was divided evenly into two observation subgroups for 1 and 7 days post cell delivery.

\section{Cardiac function by echocardiography}

All animals in Day 7 groups received cardiac function evaluation by transthoracic echocardiography prior to sacrifice (Vevo 770 ${ }^{\mathrm{TM}}$; Visual Sonic, Toronto, Canada) as described previously. ${ }^{20}$ Rats were anesthetized by isoflurane inhalation $(1.5 \%)$, with heart rate maintained at $\sim 400$ beats per minute and body temperature maintained at $37^{\circ} \mathrm{C}$ by placing rats on a heating pad. Left parasternal two-dimensional M-mode images under short-axis at the level of papillary muscles were recorded by using a $35-\mathrm{MHz}$ linear transducer. Left ventricular enddiastolic volume (LVEDV), left ventricular end-systolic volume (LVESV), left ventricular end-diastolic diameter (LVEDD) and left ventricular end-systolic diameter (LVESD) were measured. LVEF and left ventricular fractional shortening (LVFS) were calculated with standard M-mode echocardiographic equations $(\mathrm{EF}=\mathrm{LVEDV}-\mathrm{LVESV} / \mathrm{LVEDV} \times 100 ; \mathrm{FS}=\mathrm{LVEDD}-$ LVESD/LVEDD $\times 100$ ). All measurements were averaged for five consecutive cardiac cycles and performed by an experienced examiner in a blinded fashion.

\section{MSCs tracking by MRI}

Cardiac MRI was performed 1 and 7 days post-cell deliveries. MR images were obtained with a conventional cardiac-gated fast low-angle shot (FLASH) sequence on a Bruker 7.0T magnetic resonance scanner (Bruker, Madison, WI). Rats were anesthetized with $1.5 \%$ isoflurane (KeYuan, Shandong, China) in $1 \mathrm{~L} /$ minute of oxygen and monitored using a small animal instrument monitoring and gating system for respiration rate and electrocardiogram triggering. The field of view was $6 \times 6 \mathrm{~cm}$ and a $256 \times 256$ matrix was employed, yielding an in-plane resolution of $234 \times 234 \mu \mathrm{m}$. The images were acquired from successive slices along the short cardiac axis with a slice thickness of $1 \mathrm{~mm}$; 16 slices were captured from apex to base. The repetition time was $70 \mathrm{~ms}$, echo time was $2.5 \mathrm{~ms}$, and flip angle was $60^{\circ}$. The entire imaging protocol was performed in approximately 15 minutes.

\section{Optical imaging study (in vivo, ex vivo)}

The optical imaging experiments were performed using a CRi Maestro in vivo molecular imaging system (CRi, Woburn, MA) which permits coverage in the red, far-red, and NIR spectral regions. Light and cube images were acquired for in vivo or ex vivo study, respectively. After anesthetization, animals were shaved on the forelimbs, neck, chest, and abdomen and placed in a supine position. In vivo imaging was performed for each animal 1 or 7 days post delivery, followed immediately by ex vivo imaging of explanted organs (heart, lung, liver, spleen, and kidney). NIR fluorescent signal average intensities from explanted organs were quantified as counts per second per pixels by software from CRi Maestro. Parameters were set as follows: excitation wavelength coverage 595-800 nm, emission coverage 660-680 nm.

\section{Histological analysis}

After optical detection, the explanted hearts and lungs were subjected to fast-frozen sectioning for fluorescence microscopy, Prussian blue, and ED-1staining. Paraffin sections of heart tissue were prepared for Masson trichrome staining and assessed for infarct size, which was quantified as a percentage of the left ventricular transverse perimeter occupied by collagen.

\section{Data analysis}

SPSS software (v. 11.5; SPSS Inc., Chicago, IL) was used for data analysis. All data is expressed as mean \pm standard deviation of the mean for each time point. Multiple group comparisons were performed by using one-way analysis of variance; the significance of differences between two groups was analyzed by the Student's $t$-test. All tests were two-tailed and a $P$-value $<0.05$ was considered significant.

\section{Results \\ Morphological observations of cultured cells, efficiency of double labeling}

Single-nucleated cells were expanded for two passages and cells were spindle-shaped uniformly. After 14 days in culture, cells became compact and uniformly aligned in bundles with robust proliferation (Figure 1A and B). An aliquot of duallabeled cells were examined by Prussian blue staining to demonstrate the presence of intracellular SPIO. Cytoplasmic blue particles were observed and the labeling efficiency was almost 100\% (Figure 1C). All cells were labeled positive for DiD by fluorescence microscopy (Figure 1D).

\section{Effect of dual-labeling on MSC proliferation}

Cell proliferation was measured by MTT assay for both dual-labeled and unlabeled cells. As shown in Figure 1E, the difference in OD values between the two cell groups was not statistically significant $(P>0.05)$ at two time points, indicating that the dual-labeling of cells does not impart adverse effects on cell proliferation. 

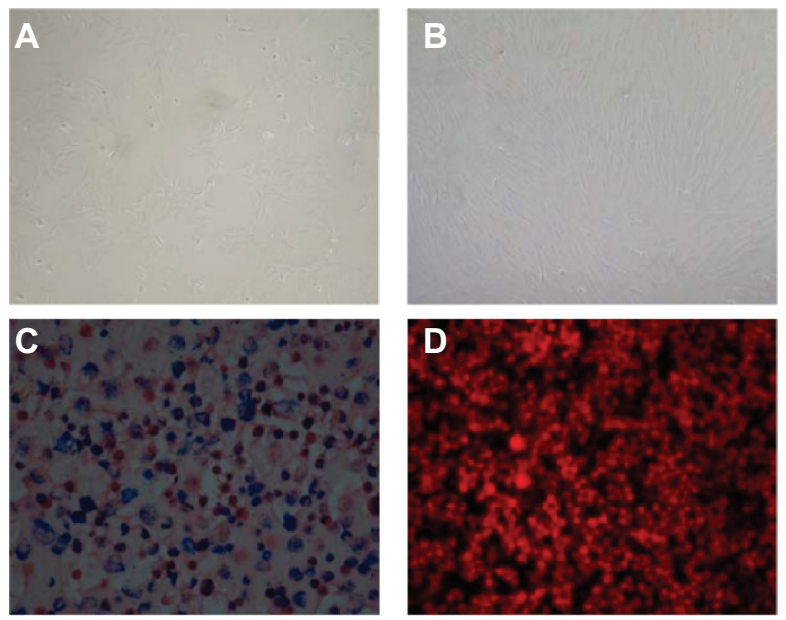

E Effect of dual-labeling on MSCs proliferation by MTT assay

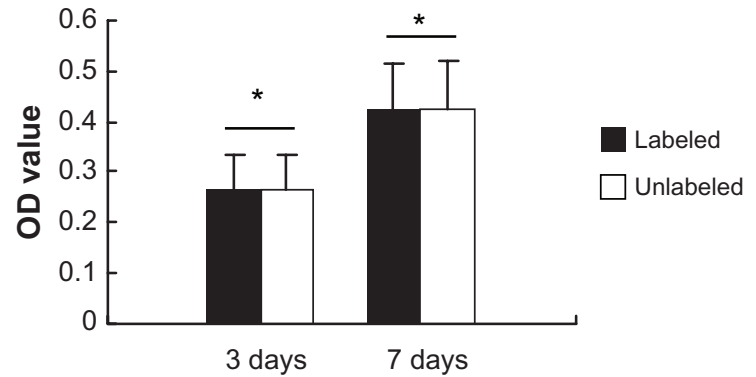

Figure I The culture and the dual-labeling of MSCs. A) At passage 2, MSCs appeared uniformly spindle-shaped $(\times 100)$. B) Cells became compact and uniformly aligned in bundles at day $14(\times 100)$. C) Prussian blue-stained cells contained cytoplasmic blue particles and SPIO-labeling efficiency was almost 100\% $(\times 100)$. D) DiD-labeled cells appear red under fluorescence microscopy ( $\times 100$; excitation wavelength $595-800 \mathrm{~nm}$, emission $660-680 \mathrm{~nm})$. E) Cell proliferation viability was measured by MTT assay for dual-labeled and unlabeled cells

Note: $* P>0.05$.

Abbreviations: MSCs, mesenchymal stem cells; SPIO, superparamagnetic iron oxide nanoparticle; DiD, I,I'-dioctadecyl-3,3,3',3'-tetramethylindodicarbocyanine, 4-chlorobenzenesulfonate salt; MTT, methyl thiazolyl tetrazolium.

\section{Correlation between cell number} and $M R T_{2}$ relaxometry (ms) or fluorescence intensity (counts)

Dual-labeled cells resuspended in algal-gel were transferred into 96-well plates at different cell concentrations. A phantom of $2 \times 10^{5}$ cells appeared black, whereas a phantom of $0.125 \times 10^{5}$ cells appeared gray. A strong correlation between the number of cells and $T_{2}$ relaxometry in the
MR images was observed $\left(R^{2}=0.9547\right.$; Figure $2 A$ and $\left.B\right)$. Fluorescence counts correlated linearly with cell number $\left(y=-0.002+0.02 x ; R^{2}=0.9976\right.$; Figure $2 C$ and $\left.D\right)$.

\section{Effect of MSC transplantation on cardiac function and infarct size after MI}

In the clinical setting, the prevention of progressive heart dysfunction as a result of myocardial infarction is
A

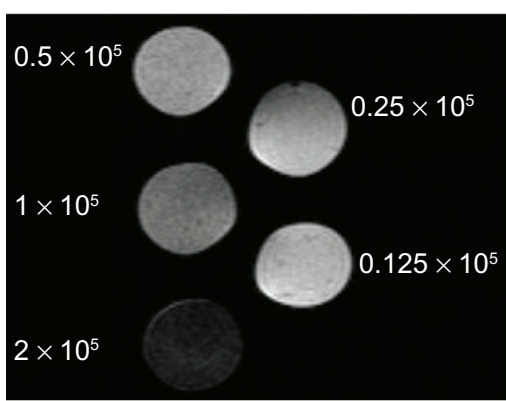

$B$

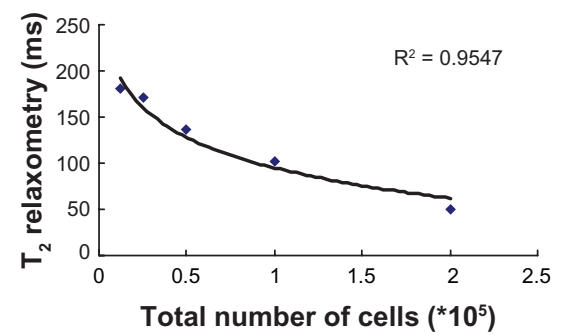

C

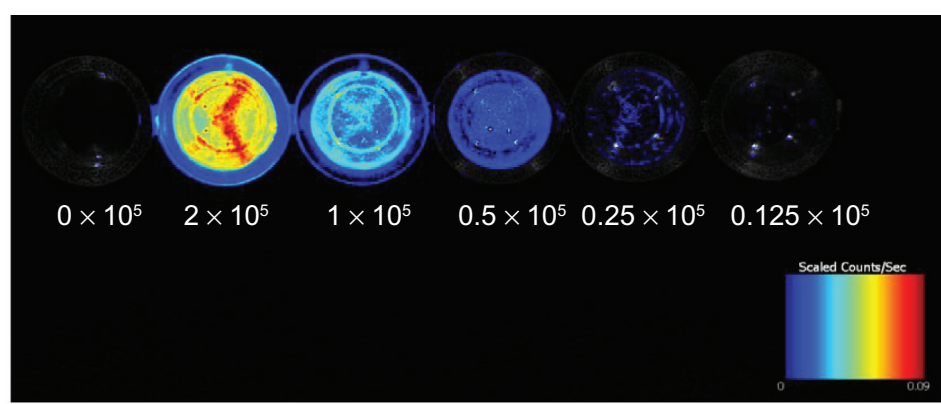

D

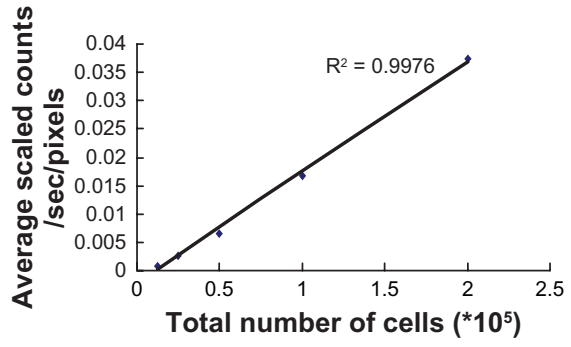

Figure 2 In vitro correlations between MR T, relaxometry or NIR fluorescent signal and the number of cells. A) A phantom of $2 \times 10^{5}$ cells appeared black, whereas a phantom of $0.125 \times 10^{5}$ cells appeared gray in the MR images. B) A strong correlation between the number of cells and $T_{2}$ relaxometry was observed ( $\left.R^{2}=0.9547\right)$. C) Fluorescent images showed that signal intensity increased with the increasing cell number. Bars represent maximum radiance. D) Correlation plot showed fluorescence counts correlated linearly with cell number $\left(y=-0.002+0.02 x ; R^{2}=0.9976\right)$.

Abbreviations: MR, magnetic resonance; NIR, near-infrared. 
of great importance. To this purpose, cardiac functional outcomes evaluated 1 week after cell delivery by echocardiography are shown in Figure 3A and Table 1. In the control and IV group, markedly decreased fractional shortening (FS) and ejection fraction (EF) compared with the IM group were observed $(P<0.01)$, corresponding to post-infarction myocardial systolic failure. However, there were no significant differences between the control and IV groups $(P>0.05)$. One week after cell transplantation, infarct size was assessed by Masson trichrome staining in each experimental group and quantified as a percentage of the left ventricular transverse perimeter occupied by collagen. While there were no differences between the control and IV group $(P>0.05)$, transplantation by IM was associated with a significant improvement in infarct size compared to the aforementioned two groups $(P<0.01$; Figure 3B and Table 1$)$.

\section{Distribution of implanted cells assessed by $\mathrm{Ol}$}

The in vivo NIR fluorescent signals of transplanted duallabeled MSCs on rat chests were evaluated in all groups by OI (Figure 4A and B). No fluorescent signals were observed in the area of the heart among control and IV groups at the two time points, but signals in IM groups last over 1 week. Quantitative analysis of the fluorescent signals in explanted hearts among two cell transplantation groups showed that the bulk of injected cells disappeared and only $2.3 \%-0.6 \%$ of cells survived from 1 to 7 days post cell delivery in the IV group (compared to the dose of transplanted cells), and approximately $23.8 \%-2.0 \%$ of transplanted cells survived in the IM group during the same time frame. The fluorescent signals of the lungs in the IV group were 5.8-2.8 fold higher than the IM group at two time points $(0.037 \pm 0.003$
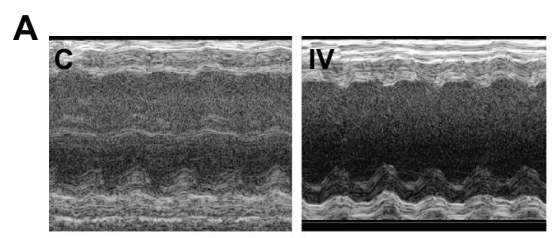

B
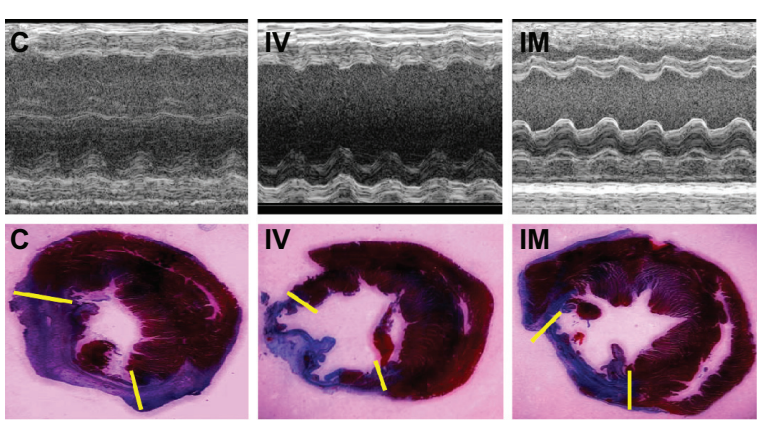

Figure 3 Measurements of cardiac function and infarct size. A) Representative M-mode images at the level of papillary muscles were recorded among three groups. B) Representative Masson's trichrome-stained histological sections to measure infarct size. Collagen presented blue but myocardium appeared red. Infarct size was quantified as the area occupied by collagen (blue; yellow hash marks).

Abbreviations: C, control group; IV, intravenous group; IM, intramyocardial group.
Table I ECHO parameters of LV cardiac function and infarct size among three groups 7 days after cell delivery

\begin{tabular}{llll}
\hline & Control (6) & IV (6) & IM (6) \\
\hline LVEF (\%) & $36.68 \pm 1.11$ & $36.62 \pm 1.18$ & $41.45 \pm 1.54^{*}$ \\
LVFS (\%) & $18.02 \pm 0.72$ & $17.98 \pm 0.71$ & $20.51 \pm 0.84^{*}$ \\
Infarct size (\%) & $41.12 \pm 3.3$ & $40.92 \pm 3.27$ & $23.10 \pm 1.98 *$ \\
\hline
\end{tabular}

Notes: Data represented as mean \pm SD. $* P<0.0$ I, IM group compared with IV group and control group. Infarct size was quantified as a percentage of the LV transverse perimeter occupied by collagen.

Abbreviations: $\mathrm{ECHO}$, echocardiography; LV, left ventricular; LVEF, left ventricular ejection fraction; FS, fractional shortening; C, control group; IV, intravenous group; IM, intramyocardial group; SD, standard deviation.

vs $0.006 \pm 0.001 ; 0.014 \pm 0.003$ vs $0.005 \pm 0.001$, respectively, $P<0.01$ ) (Figure $5 \mathrm{~A}-\mathrm{C}$ ).

\section{Monitoring (tracking) implanted cells by MRI}

In the IM group, labeled cells were visualized in the infarcted myocardium as hypointense areas by serial MRI studies. While signal size decreased from day 1 to day 7, no signal was observed in IV group (Figure 4A and B).

\section{Histological examination}

Fast-frozen sections of the myocardium and the lung in cell transplantation groups were prepared for Prussian blue staining and fluorescent detection. Blue SPIO particles and NIR fluorescent signals were observed in the myocardial section of the IM group at both time points. In contrast, SPIO particles and fluorescent signals were found only in the lungs of the IV group. Interestingly, a mass of transplanted cells were entrapped in the pulmonary interstitium (Figure 6A and B). Neither blue particles nor fluorescent signals were detected in the myocardium from the IV group (data not shown). Prussian blue and ED-1 immunohistochemical costaining of the myocardium from the IM group 7 days post transplantation showed a distribution of blue particles nearly consistent with ED-1 immunohistochemical bright field staining, indicating that most implanted stem cells were phagocytosed by macrophages (Figure 6C).

\section{Discussion}

In the current study, we found that highly efficient duallabeling of MSCs does not have adverse effects on cell proliferation over the course of 1 week. Further, direct IM injection of MSCs could increase the cell engraftment and significantly improve ventricular contractility post MI.

There are controversial results regarding implantation efficiency and improved function in cardiac cell therapy. These discrepancies may arise from the different modes of 


\section{NIR optical imaging}
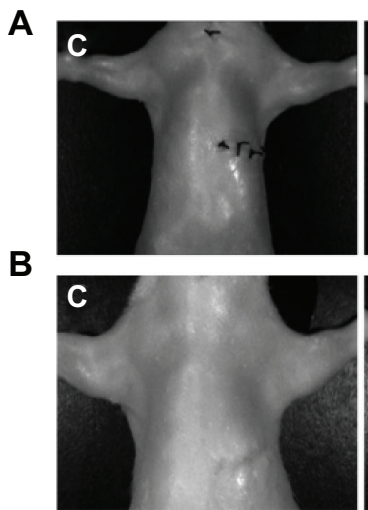
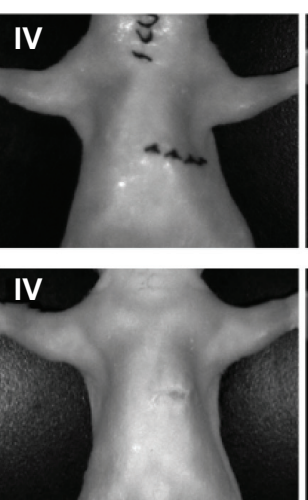
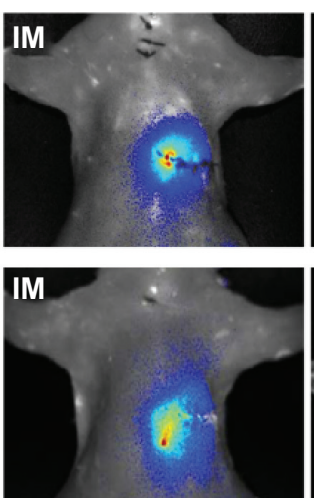

MR imaging
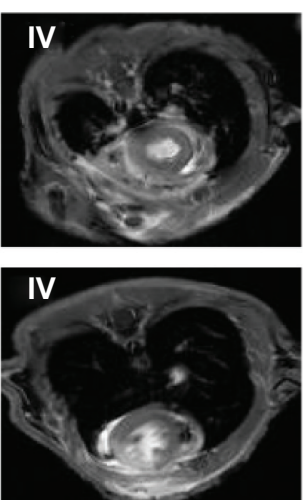

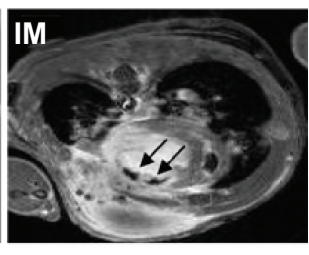

IM

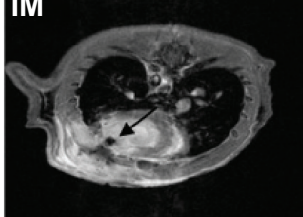

Figure 4 The in vivo NIR fluorescent signal and MR detections. No fluorescent signals were observed in the heart areas of control and IV groups at two time points, but the signals in IM groups lasted over I week. MR imaging showed that SPIO-labeled cells were visualized in the infarcted myocardium as hypointense areas by serial MRI studies from day I to day 7 in the IM group, but no signals were found in the IV group. A) One day after cell delivery. B) Seven days after cell delivery.

Abbreviations: NIR, near-infrared; MR, magnetic resonance; SPIO, superparamagnetic iron oxide; C, control group; IV, intravenous group; IM, intramyocardial group.

cell delivery. To our knowledge, this is the first report of combined MR and NIR optical imaging techniques to explore the efficacy of two cell delivery routes in a rat model of MI.

The development of molecular imaging techniques has allowed visualization of stem cell homing, migration, location, and proliferation. ${ }^{21}$ Although there are no current ideal imaging modalities for in vivo stem cell tracking, each individual imaging modality presents unique advantages and disadvantages. Thus, multimodality may provide a useful means for stem cell tracking. ${ }^{22}$ Imaging by high-field MR
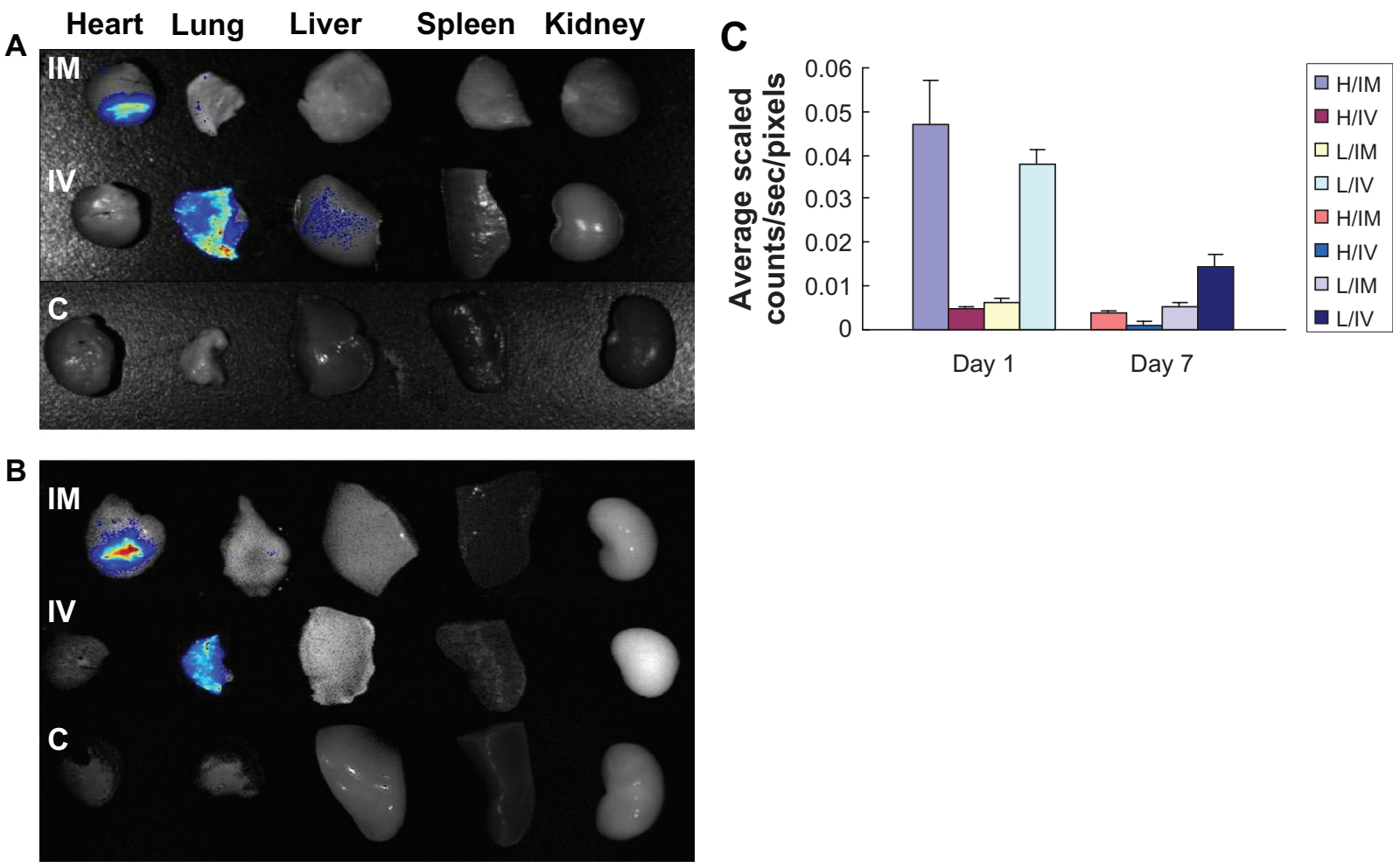

Figure 5 Ex vivo optical imaging study. A-B) Representative NIR fluorescent images in explanted organs I or 7 days after labeled MSCs were implanted into rats by means of intravenous or intramyocardial delivery I day after coronary artery ligation. C) Quantitative analysis of NIR fluorescent signals in explanted hearts and lungs among the two cell transplanted groups, indicating that cell delivery by direct IM injection resulted in a 3-I0-fold higher fluorescent signal than IV infusion in explanted hearts from day I to day 7. In contrast, compared with the IM group, NIR fluorescent signals exist in lungs from the IV group during I week post cell delivery. (A: One day after cell delivery. B: Seven days after cell delivery).

Abbreviations: NIR, near-infrared; H, heart; L, lung; C, control group; IV, intravenous group; IM, intramyocardial group. 


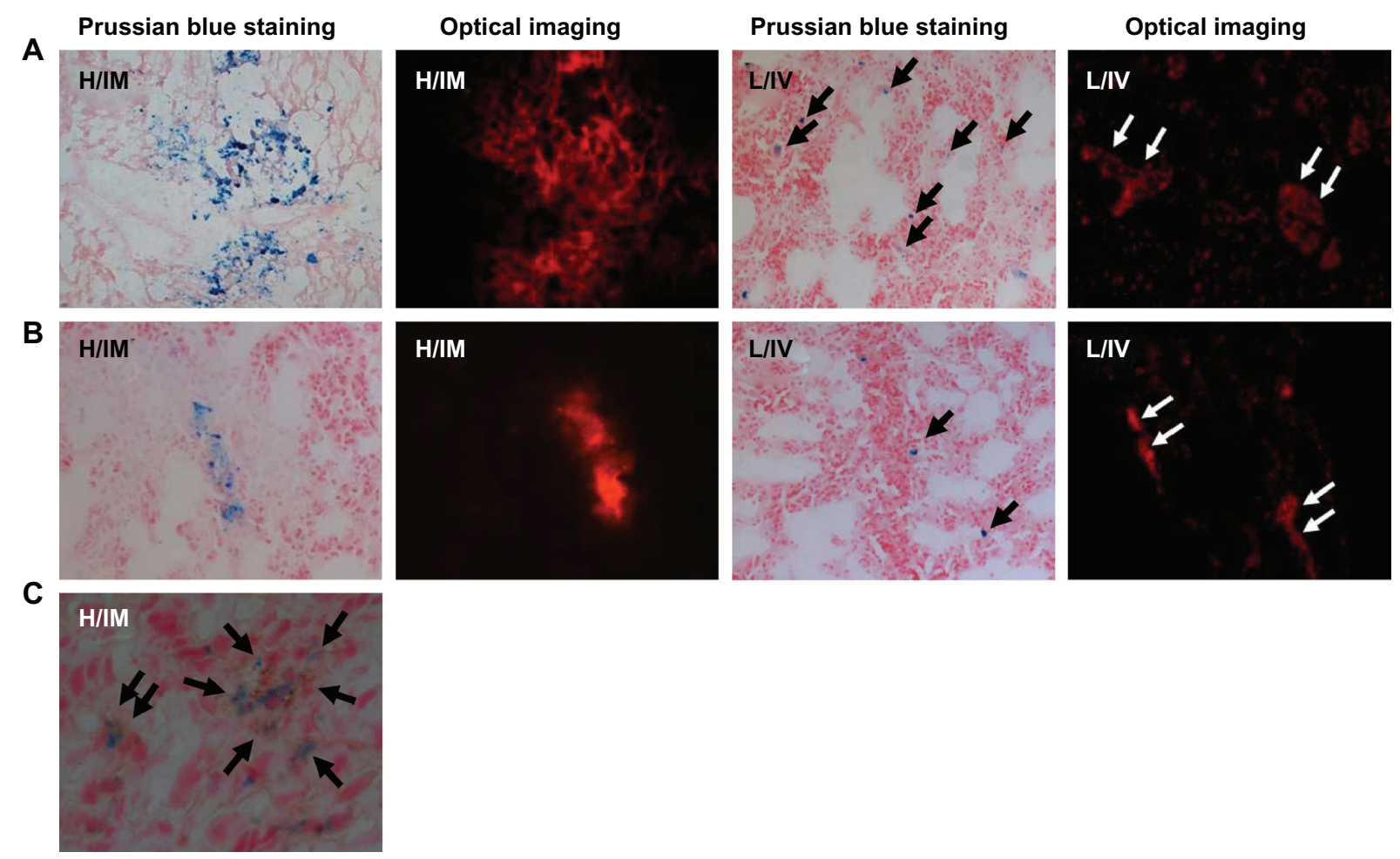

Figure 6 Cryosections of the myocardium and the lung were detected by Prussian blue staining, fluorescence microscopy, and immunohistochemical staining. A-B) SPIO blue particles and NIR fluorescent signals were observed in the myocardium of the IM group at two time points. However, in the IV group, SPIO particles and fluorescent signals were found only in the lungs. Interestingly, a mass of transplanted cells were located in pulmonary interstitium. $(\times 100 ; A$ : One day after cell delivery, B: Seven days after cell delivery). C) Section from infarcted myocardium in the IM group at day 7 was determined by Prussian blue and ED-I immunohistochemical costaining. The distribution of blue particles was consistent with ED-Istaining, indicating that most implanted stem cells were phagocytosed by macrophages $(\times 400)$.

Abbreviations: SPIO, superparamagnetic iron oxide; NIR, near-infrared; H, heart; L, lung; IV, intravenous group; IM, intramyocardial group.

scanners enables us to study rodent cardiac structure and noninvasively track stem cells in an infarcted heart. ${ }^{23}$ Using a 7.0-Tesla scanner to identify transplanted cells by MR, we identified the IM injection sites of iron-labeled MSCs as dark spots in vivo. The signal size faded during 1-week follow-up, indicating that the transplanted cells were gradually lost in heart tissue. However, imaging by MR has low sensitivity and fails to quantify the number of engrafted cells. ${ }^{24}$ In parallel, using the proven highly sensitive optical imaging for in vitro cell labeling and in vivo cell tracking, ${ }^{25}$ we observed that MSCs could be labeled efficiently by $\mathrm{DiD}$ in vitro and fluorescence counts correlated linearly with cell number increase, allowing cell engraftment to be assessed in explanted hearts. Ex vivo studies showed that more MSCs were engrafted into the myocardium in the IM group compared to the IV group. Conversely, a majority of cells were entrapped in the lungs of the IV group. These findings are in agreement with previous studies describing that very few stem cells homed to infarcted myocardium through IV infusion. ${ }^{26,27}$

IV infusion and IM injection are currently the predominant stem cell delivery routes for the injured heart of small animals. Each transplant route attempts to maximize not only the ease of administration, but the targeted homing, retention, stem cell engraftment to damaged myocardium and improved cardiac function. ${ }^{28}$ The therapeutic effect(s) of MSCs relies on their capacity to engraft and survive in distinctive target tissue, and poor stem cell survival is responsible for unsatisfactory cell transplantation prognosis post MI. ${ }^{29}$ Compared to IV infusion, cell delivery by IM injection increased the engraftment and survival of MSCs in infarcted hearts. These results might explain why cell delivery by the IM route could ameliorate the cardiac function and infarct size post MI, rather than by IV infusion.

The precise mechanisms of the beneficial effects of transplanted MSCs are uncertain. Our recent research suggested that the major mechanism of functional benefits appears to be paracrine pathways of the engrafted EPCs (endothelial progenitor cells) post MI, which facilitates endogenous repair processes..$^{30}$ The targets of these paracrine effects most likely include both mature cells and resident progenitor cells in the recipient heart, which can induce neovascularization, inhibit apoptosis, reduce infarct size and scar formation, and improve myocardial contractility. ${ }^{31,32}$ Although costaining of iron and ED-1 in myocardium showed that most transplanted cells had 
been phagocytosed by macrophages in the infarcted heart on day 7 , the OI detection from cryosections and explanted hearts in the IM group indicated that some remaining implanted MSCs survived or may have differentiated into other cardiac cell lineages, as described by the most recent findings of Tsuji and colleagues describing transdifferentiation of MSC application in the infarcted myocardium. ${ }^{33}$

The rats used in this study were low in weight, and a low dose of transplanted cells $\left(1 \times 10^{6}\right.$ per rat $)$ was applied. We speculate that the administration of a higher dose of cells or genetically modified stem cells may produce better outcomes despite IV or IM delivery route. Regardless, these data indicate that the optimal transplantation route likely plays an important role in cardiac cell therapy and the IM route may provide an advantage over IV infusion for retention of stem cells and cardiac function protection.

\section{Acknowledgment}

This work was supported by the National Nature Science Foundation of the People's Republic of China (No. 30871071, No.81070085) and (No. 7790000004, No.091028653).

\section{Disclosure}

The authors report no conflicts of interest in this work.

\section{References}

1. Lasala GP, Minguell JJ. Bone marrow-derived stem/progenitor cells: their use in clinical studies for the treatment of myocardial infarction. Heart Lung Circ. 2009;18(3):171-180.

2. Jain M, Pfister O, Roger J, Hajjar RJ, Liao R. Mesenchymal stem cells in the infarcted heart. Coron Artery Dis. 2005;16(2):93-97.

3. Wei HM, Wong P, Hsu LF, Shim W. Human bone marrow-derived adult stem cells for post-myocardial infarction cardiac repair: current status and future directions. Singapore Med J. 2009;50(10):935-942.

4. Janssens S, Dubois C, Bogaert J, et al. Autologous bone marrow derived stem-cell transfer in patients with ST-segment elevation myocardial infarction: double-blind, randomised controlled trial. Lancet. 2006;367(9505):113-121.

5. Lunde K, Solheim S, Aakhus S, et al. Intracoronary injection of mononuclear bone marrow cells in acute myocardial infarction. $N$ Engl J Med. 2006;355(12):1199-1209.

6. Penicka M, Horak J, Kobylka P, et al. Intracoronary injection of autologous bone marrow-derived mononuclear cells in patients with large anterior acute myocardial infarction. J Am Coll Cardiol. 2007;49(24):2373-2374.

7. Bel A, Messas E, Agbulut O, et al. Transplantation of autologous fresh bone marrow into infarcted myocardium: a word of caution. Circulation. 2003;108(II):247-252.

8. Yamahara K, Nagaya N. Mesenchymal stem cells for the treatment of heart disease. Regen Med. 2007;2(2):107-109.

9. Pittenger MF, Martin BJ. Mesenchymal stem cells and their potential as cardiac therapeutics. Circ Res. 2004;95(1):9-20.

10. Schuleri KH, Boyle AJ, Hare JM. Mesenchymal stem cells for cardiac regenerative therapy. Handb Exp Pharmacol. 2007;(180):195-218.
11. Price MJ, Chou CC, Frantzen M, et al. Intravenous mesenchymal stem cell therapy early after reperfused acute myocardial infarction improves left ventricular function and alters electrophysiologic properties. Int J Cardiol. 2006;111(2):231-239.

12. Wang T, Tang W, Sun S, Ristagno G, Huang L, Weil MH. Intravenous infusion of bone marrow mesenchymal stem cells improves myocardial function in a rat model of myocardial ischemia. Crit Care Med. 2007;35(11):2587-2593.

13. Li Q, Turdi S, Thomas PD, Zhou T, Ren J. Intra-myocardial delivery of mesenchymal stem cells ameliorates left ventricular and cardiomyocyte contractile dysfunction following myocardial infarction. Toxicol Lett. 2010;195(2-3):119-126.

14. Tang YL, Zhu W, Cheng M, et al. Hypoxic preconditioning enhances the benefit of cardiac progenitor cell therapy for treatment of myocardial infarction by inducing CXCR4 expression. Circ Res. 2009;104(10):1209-1216.

15. Carr CA, Stuckey DA, Tatton L, et al. Bone marrow-derived stromal cells home to and remain in the infarcted rat heart but fail to improve function: an in vivo cine-MRI study. Am J Physiol Heart Circ Physiol. 2008;295(2):H533-H542.

16. Wei H, Ooi TH, Tan G, et al. Cell delivery and tracking in postmyocardial infarction cardiac stem cell therapy: an introduction for clinical researchers. Heart Fail Rev. 2010;15(1):1-14.

17. Tousoulis D, Briasoulis A, Antoniades C, et al. Heart regeneration: what cells to use and how? Curr Opin Pharmacol. 2008;8(2):211-218.

18. Barbash IM, Chouraqui P, Baron J, et al. Systemic delivery of bone marrow-derived mesenchymal stem cells to the infarcted myocardium: feasibility, cell migration, and body distribution. Circulation. 2003; 108(7):863-868.

19. Kraitchman DL, Heldman AW, Atalar E, et al. In vivo magnetic resonance imaging of mesenchymal stem cells in myocardial infarction. Circulation. 2003;107(18):2290-2293.

20. Zhu W, Shou W, Payne RM, Caldwell R, Field LJ. A mouse model for juvenile doxorubicin-induced cardiac dysfunction. Pediatr Res. 2008;64(5):488-494.

21. Swijnenburg RJ, Van der Bogt KEA, Sheikh AY, Cao F, Wu JC. Clinical hurdles for the transplantation of cardiomyocytes derived from human embryonic stem cells: role of molecular imaging. Curr Opin Biotechnol. 2007;18(1):38-45.

22. Hoshino K, Ly HQ, Frangioni JV, Hajjar RJ. In vivo tracking in cardiac stem cell-based therapy. Prog Cardiovasc Dis. 2007;49(6):414-420.

23. Tyler DJ, Lygate CA, Schneider JE, Cassidy PJ, Neubauer S, Clarke K. CINE-MR imaging of the normal and infarcted rat heart using an 11.7 T vertical bore MR system. J Cardiovasc Magn Reson. 2006; $8(2): 327-333$

24. Ebert SN, Taylor DG, Nguyen HL, et al. Noninvasive tracking of cardiac embryonic stem cells in vivo using magnetic resonance imaging techniques. Stem Cells. 2007;25(11):2936-2944.

25. Sutton EJ, Henning TD, Pichler BJ, Bremer C, Daldrup-Link HE. Cell tracking with optical imaging. Eur Radiol. 2008;18(10):2021-2032.

26. Aicher A, Brenner W, Zuhayra M, et al. Assessment of the tissue distribution of transplanted human endothelial progenitor cells by radioactive labeling. Circulation. 2003;107(16):2134-2139.

27. Freyman T, Polin G, Osman H, et al. A quantitative, randomized study evaluating three methods of mesenchymal stem cell delivery following myocardial infarction. Eur Heart J. 2006;27(9):1114-1122.

28. LaPar DJ, Kron IL, Yang Z. Stem cell therapy for ischemic heart disease: where are we? Curr Opin Organ Transplan. 2009;14(1):79-84.

29. Zhang H, Chen H, Wang W, et al. Cell survival and redistribution after transplantation into damaged myocardium. J Cell Mol Med. 2010;14(5):1078-1082.

30. Yao Y, Li Y, Ma G, et al. In vivo magnetic resonance imaging of injected endothelial progenitor cells after myocardial infarction in rats. Mol Imaging Bio. 2010. [Epub ahead of print]. 
31. Crisostomo PR, Wang M, Markel TA, et al. Stem cell mechanisms and paracrine effects: potential in cardiac surgery. Shock. 2007; 28(4):375-383.

32. Nakanishi C, Yamagishi M, Yamahara K, et al. Activation of cardiac progenitor cells through paracrine effects of mesenchymal stem cells. Biochem Biophys Res Commun. 2008;374(1):11-16.
33. Tsuji H, Miyoshi S, Ikegami Y, et al. Xenografted human amniotic membrane-derived mesenchymal stem cells are immunologically tolerated and transdifferentiated into cardiomyocytes. Circ Res. 2010;106(10):1613-1623.

\section{Publish your work in this journal}

The International Journal of Nanomedicine is an international, peerreviewed journal focusing on the application of nanotechnology in diagnostics, therapeutics, and drug delivery systems throughout the biomedical field. This journal is indexed on PubMed Central,

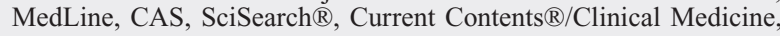

Journal Citation Reports/Science Edition, EMBase, Scopus and the Elsevier Bibliographic databases. The manuscript management system is completely online and includes a very quick and fair peer-review system, which is all easy to use. Visit http://www.dovepress.com/ testimonials.php to read real quotes from published authors.

Submit your manuscript here: http://www.dovepress.com/international-journal-of-nanomedicine-journal 